\title{
Quantum percolation in two-dimensional antiferromagnets
}

\author{
Rong Yu, ${ }^{1,2}$ Tommaso Roscilde, ${ }^{1}$ and Stephan Haas ${ }^{1}$ \\ ${ }^{1}$ Department of Physics and Astronomy, University of Southern California, Los Angeles, CA 90089-0484 \\ ${ }^{2}$ Kavli Institute for Theoretical Physics, University of California, Santa Barbara, CA 93106-4030
}

\begin{abstract}
The interplay of geometric randomness and strong quantum fluctuations is an exciting topic in quantum many-body physics, leading to the emergence of novel quantum phases in strongly correlated electron systems. Recent investigations have focused on the case of homogeneous site and bond dilution in the quantum antiferromagnet on the square lattice, reporting a classical geometric percolation transition between magnetic order and disorder. In this study we show how inhomogeneous bond dilution leads to percolative quantum phase transitions, which we have studied extensively by quantum Monte Carlo simulations. Quantum percolation introduces a new class of two-dimensional spin liquids, characterized by an infinite percolating network with vanishing antiferromagnetic order parameter.
\end{abstract}

PACS numbers: 75.10.Jm, 75.10.Nr, 75.40.Cx, 64.60.Ak

Percolation occurs in a variety of contexts, 1] ranging from blood vessel formation to clusters of atoms deposited on substrate surfaces. A fundamental question is whether the classical picture of permeating networks [2] applies as well to strongly fluctuating quantum systems. In this respect, low-dimensional quantum magnets offer an ideal playground to probe the interplay between quantum fluctuations and geometric randomness both theoretically and experimentally $[3,4]$.

Two electronic spins coupled by an antiferromagnetic Heisenberg interaction, $\mathcal{H}=J \boldsymbol{S}_{1} \cdot \boldsymbol{S}_{2}(J>0)$, combine into a singlet state with purely quantum character. Such singlets are the fundamental building blocks of many novel phases in quantum antiferromagnets. For instance, the ground state of a Heisenberg ladder is a coherent superposition of singlet coverings involving mostly nearestneighbor spins. This resonating valence bond (RVB) state [6] is realized in a number of antiferromagnetically correlated materials for which magnetometry and neutron. scattering measurements indicate the absence of magnetic order down to the lowest accessible temperatures 7]. In contrast, the $S=1 / 2$ Heisenberg antiferromagnet on the square lattice has an antiferromagnetically long-range-ordered ground state [8]. However, as shown. in Fig. 1(A), a square lattice can be decomposed into dimers and ladders in such a way that there are no two adjacent dimers/ladders. This suggests that, if lattice disorder can isolate some of these substructures, it may lead to quantum disordered ground states.

Recently, the effect of site dilution on the squarelattice $S=1 / 2$ quantum Heisenberg antiferromagnet has been probed experimentally in the compound $\mathrm{La}_{2} \mathrm{Cu}_{1-p}(\mathrm{Zn}, \mathrm{Mg})_{p} 0_{4}$, in which magnetic $\mathrm{Cu}^{2+}$ ions are replaced randomly by non-magnetic $\mathrm{Zn}^{2+}$ or $\mathrm{Mg}^{2+}$ ions [3]. The pure system $(p=1)$ displays long-range antiferromagnetic order [8]. Upon doping with static nonmagnetic impurities, the order parameter is reduced until it vanishes at a critical doping concentration $p_{c}$. Both experimental data [3] and numerical simulations [3, 4] indicate a magnetic transition at the geometric percolation threshold $p^{*}=0.40725$ [2] beyond which the system breaks up into finite clusters. An analogous result holds for the case of bond dilution, where a fraction $p$ of bonds is cut at random in the system: again the onset of disorder is observed at the percolation threshold $p_{c}=p^{*}=0.5[4]$. In both cases this implies that the magnetic transition is controlled by the underlying geometric transition. A completely different picture of the
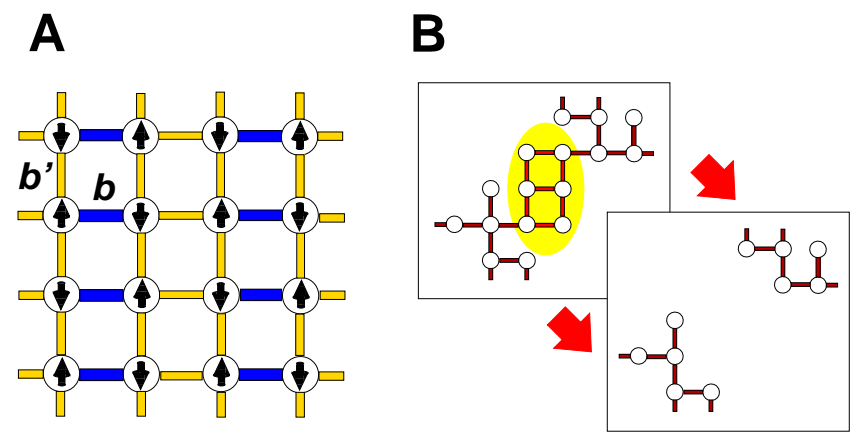

FIG. 1: (color online) (A) A square lattice quantum antiferromagnet composed of dimers (blue bonds, b) and ladders (yellow bonds, b'). (B) A segment of ladder in the percolating cluster leads to local RVB states (yellow ellipsis). The spins involved in the RVB state are very weakly correlated to the rest of the cluster, causing an effective fragmentation of the percolating cluster into non-percolating subclusters.

effect of disorder might instead emerge when resonating valence bonds dominate the ground state of the system. In fact, the spins involved in a local RVB/singlet state are weakly correlated to the remainder of the system, so that, from the point of view of magnetic correlations, we can roughly consider these spins missing from the geometric cluster they belong to, as sketched in Fig. 1(B). This implies that the percolating cluster is effectively fragmented by the formation of RVB/singlet states, and therefore 
the magnetic transition is hindered with respect to the geometric transition, acquiring in turn the nature of a quantum phase transition. Nonetheless, according to this argument the magnetic quantum transition appears as a renormalized percolative transition, with an effective geometry of the clusters dictated by quantum effects. This scenario is referred to as quantum percolation.

In this work we investigate the occurrence of quantum percolation in the $S=1 / 2$ quantum antiferromagnet on the square lattice with inhomogeneous bond dilution, modeled by the Hamiltonian

$$
\mathcal{H}=\sum_{b} J_{b} \boldsymbol{S}_{1, b} \cdot \boldsymbol{S}_{2, b}+\sum_{b^{\prime}} J_{b^{\prime}} \boldsymbol{S}_{1, b^{\prime}} \cdot \boldsymbol{S}_{2, b^{\prime}}
$$

Here $\boldsymbol{S}$ are $S=1 / 2$ spin operators, the bonds $b$ are dimer bonds in Fig. 1(B), and the bonds $b^{\prime}$ are the inter-dimer bonds. $J_{b}, J_{b^{\prime}}>0$ are random antiferromagnetic couplings drawn from a bimodal distribution taking the two values $J$ ('on') and 0 ('off'), with different probabilities of the 'on'-state, $p\left(J_{b}=J\right)=P$ and $p\left(J_{b^{\prime}}=J\right)=P^{\prime}$. In the special case $P=P^{\prime}$ the homogeneous bond-dilution problem is recovered: the percolation threshold coincides with a magnetic order-disorder transition at the classical critical value $P_{c}=P_{c}^{\prime}=0.5[2]$. In contrast, for $P \neq P^{\prime}$ one encounters inhomogeneous bond-dilution. In particular, at $P=1$ the system is an array of randomly coupled dimers, and in the opposite limit $P^{\prime}=1$ the system is a set of randomly coupled ladders.

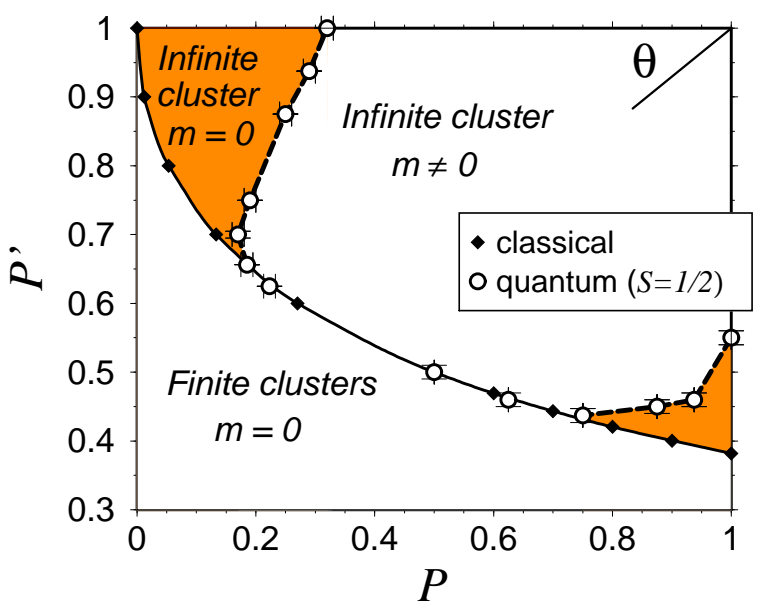

FIG. 2: Phase diagram of the inhomogeneously bond-diluted $S=1 / 2$ antiferromagnet on the square lattice. The colored area indicates the quantum-disordered region in which the system has developed an infinite percolating cluster, but the magnetization $m$ of the system vanishes because of quantum fluctuations. The angle $\theta$ parametrizes the critical curve.

We determine the geometric percolation threshold for the inhomogeneous bond-dilution problem by generalizing a very efficient algorithm recently developed for homogeneous percolation [9]. The resulting phase boundary is shown with filled diamond symbols in Fig. 2] A continuous transition line, with constant critical exponents of

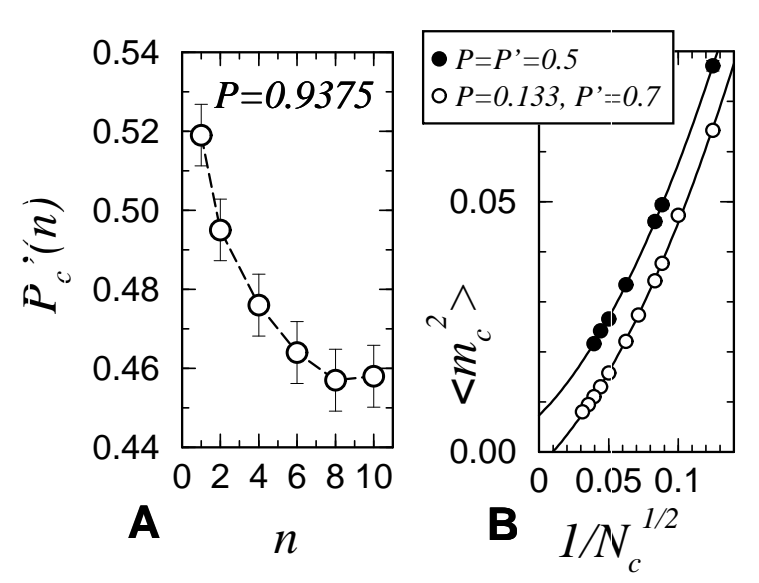

FIG. 3: (A) Estimates of the quantum critical point as the value of the disorder showing the critical scaling $\xi_{x}, \xi_{y} \sim L$ at a temperature $T=J / n L$, plotted as a function of $n$. For $n=$ 8 the estimate clearly becomes temperature-independent. (B) Disorder-averaged square order parameter on the percolating cluster only as a function of the size $N_{c}$ of the percolating cluster for the homogeneous percolation point $\left(P=P^{\prime}=\right.$ $0.5)$ and for a classical inhomogeneous percolation point $(P=$ $\left.0.133, P^{\prime}=0.7\right)$. The temperature is $T=1 /\left(8 N_{c}\right)$. The solid lines are cubic polynomial fits.

2D percolation [2], is found, connecting the percolation threshold for randomly coupled dimers, $\boldsymbol{P}=\left(P, P^{\prime}\right)=$ $(1,0.382)$ with the percolation point $\boldsymbol{P}=(1 / 2,1 / 2)$ of the homogenously diluted systems, and terminating at the critical point for randomly coupled ladders, $\boldsymbol{P}=$ $(0,1)$. In this limiting case an infinitesimal concentration of dimer bonds is sufficient to create a percolating cluster in the system.

When classical spins $(S=\infty)$ are placed on each lattice site, long-range antiferromagnetic order follows trivially the onset of geometric percolation. In contrast, this picture changes drastically for $S=1 / 2$ quantum spins. Approaching the two limits of randomly coupled dimers $(P \rightarrow 1)$ and ladders $\left(P^{\prime} \rightarrow 1\right)$, the formation of singlet states for the spins on the dimers ( $b$-bonds) or on the ladders ( $b^{\prime}$-bonds) are favored statistically by geometry, such that quantum fluctuations are strongly enhanced. This contrasts with the case of homogeneous bond percolation, $P=P^{\prime}$, in which quantum effects do not change the classical picture 4]. Therefore the crossover from homogeneous to inhomogeneous percolation in the $S=1 / 2$ antiferromagnet is accompanied by a continuous enhancement of quantum fluctuations.

To determine the magnetic phase diagram in the quantum limit we use the Stochastic Series Expansion Quantum Monte Carlo (QMC) method based on the directedloop algorithm 10]. This technique enables us to access ultra-low temperatures on large $L \times L$ lattices whose size is well inside the scaling range of the critical regime. To obtain finite-size scaling at $T \rightarrow 0$ we have carried out simulations on lattices as large as $L=64$. For 
strongly inhomogeneous systems, we have studied the $T \rightarrow 0$ behavior by sitting at an inverse temperature $\beta J=8 L$, at which the analysis of critical scaling leads to a temperature-independent estimate of the transition point, as shown in Fig. 3(A). For systems closer to the homogeneous limit we found necessary to cool the system to even lower temperatures, which we can efficiently reach by a $\beta$-doubling approach [4]. Finite temperature properties are studied on larger clusters with $L=200$. Furthermore, simulations are also carried out at the percolation threshold on percolating clusters of exact size $N_{c}$ grown freely from an initial seed (see Ref. 4] for a similar analysis). For each value of $P$ and $P^{\prime}$ we average over $100 \div 300$ different realizations of the bond disorder (disorder averages are indicated as $\langle\ldots\rangle)$. The finite staggered magnetization $\sqrt{\left\langle m^{2}\right\rangle}=\sqrt{3\langle S(\pi, \pi)\rangle / L^{2}}$ of an antiferromagnetically ordered ground state is accompanied by a divergent spin-spin correlation length along the $x$ and $y$ direction, $\xi_{x}$ and $\xi_{y}$. The location of the magnetic transition is obtained from the bond concentration at which the $T=0$ correlation lengths diverge linearly with lattice size, $\xi_{x}, \xi_{y} \sim L$.

The transition line for the quantum antiferromagnet and for the classical percolation problem are clearly different. This shows that the magnetic phase transition in the case of inhomogeneous bond dilution turns into a quantum phase transition for sufficiently strong inhomogeneities, leaving the percolation transition line at two multicritical points. In particular, between the two transition lines there is an intermediate regime (colored area) in which geometrical percolation has been reached, such that there is an infinite percolating cluster [2]. Nonetheless, magnetic order is absent, as shown by the scaling of the order parameter in Fig. 3(B). Here the disorderaveraged squared magnetization $m_{c}$ of the percolating cluster only is shown as a function of the cluster size. Unlike the case of the homogeneous percolation point [4] $P=P^{\prime}=0.5$, the percolating cluster in the strongly inhomogeneous case shows a vanishing magnetization at the onset of geometric percolation, so that the addition of extra bonds is required for the magnetic order to set in. The quantum disordered regime of the phase diagram corresponds to an infinite family of two-dimensional quantum spin liquids for which the antiferromagnetic Heisenberg interaction does not lead to spontaneous symmetry breaking in the thermodynamic limit.

Moreover, the phase diagram in Fig. 2 shows remarkable reentrant behavior. Decreasing $P\left(P^{\prime}\right)$ from 1 to 0 with fixed $P^{\prime}(P)$ in the range $0.45 \lesssim P^{\prime} \lesssim 0.55$ $(0.2 \lesssim P \lesssim 0.32)$, the system experiences first a magnetic transition from the spin liquid to the ordered antiferromagnetic state. This is a clean example of the order-bydisorder mechanism, in which magnetic order is induced by randomness due to the geometric destruction of local RVB/singlet states.

Unlike in other doped lattices [1], we do not ob- serve the occurrence of residual magnetic order due to the long-range effective interactions [12] between spins not involved in a local RVB/singlet state. We ascribe this finding to a distinctive feature of bond dilution as opposed to other forms of disorder: in fact, when the cut of a bond does not lead to the local enhancement of quantum fluctuations, it leaves always two nearby spins not forming a singlet with their neighbors, so that each unpaired spin finds immediately its partner to build a longer-range singlet.
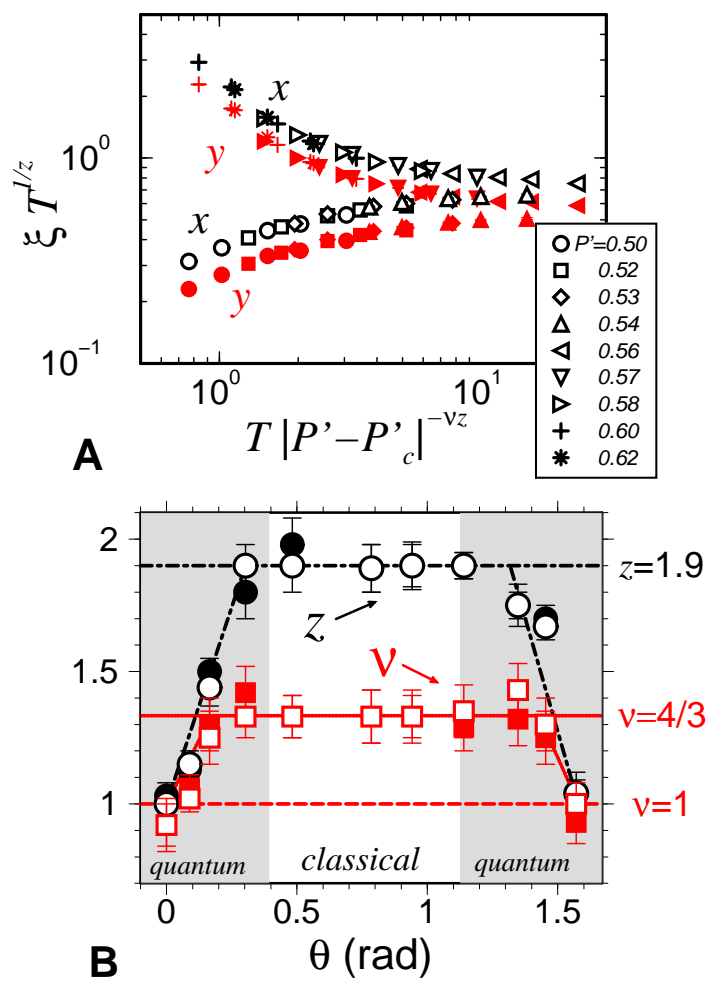

FIG. 4: (A) Scaling plot of $\xi_{x}$ (open symbols), $\xi_{y}$ (full symbols) for $P=1$ (randomly coupled dimers). (B) Critical exponents $z$ and $\nu$ for the magnetic transition in the inhomogeneously bond-diluted $\mathrm{S}=1 / 2$ Heisenberg antiferromagnet. The angle $\theta$ parametrizes the critical line in Fig. 2 Open (full) symbols refer to estimates obtained through the scaling of $\xi_{x}\left(\xi_{y}\right)$. The shaded areas correspond to the regions of parameters in which the transition is quantum. All lines are guides to the eye.

What are the critical exponents associated with this quantum phase transition? The finite-temperature quantum critical behavior of the correlation length $\xi \sim T^{-1 / z}$ determines the dynamical critical exponent $z$ [5, 13], whereas the critical exponent $\nu$ governs the divergence of the correlation length at the $T=0$ critical point, $\xi \sim\left|\boldsymbol{P}-\boldsymbol{P}_{c}\right|^{-\nu} . \quad \boldsymbol{P}_{c}$ can be obtained as the value of disorder at which $\xi$ exhibits a power-law behavior as a function of $T$, and it is in agreement with the estimates coming from the $T=0$ analysis described above. From the scaling relation

$$
\xi(\boldsymbol{P} ; T) \sim \frac{1}{T^{1 / z}} F_{\xi}\left(T\left|\boldsymbol{P}-\boldsymbol{P}_{c}\right|^{\nu z}\right)
$$


the exponent $\nu$ can be determined as the one realizing the best collapse of $T^{1 / z} \xi(\boldsymbol{P} ; T)$ curves plotted versus $T\left|\boldsymbol{P}-\boldsymbol{P}_{c}\right|^{\nu z}$ for different $\boldsymbol{P}$ values, as shown in Fig. 过(A). The values extracted for $\nu$ and $z$ are shown in Fig. 廿(B) as a function of the angle $\theta=\tan ^{-1}\left[\left(1-P^{\prime}\right) /(1-P)\right]$ which parametrizes the critical curve of Fig. 2] The $\nu$ exponent is found to satisfy the Harris criterion for disordered systems 15] $\nu \geq 2 / d=1$ within error bars. Moreover, we observe that $z$ and $\nu$ are almost constant over a large portion of the critical line, taking the values $z \approx 1.9$ and $\nu \approx 4 / 3$, and they both show a drop to $z, \nu \approx 1$ only near the edges of the critical line in the strongly quantum regimes $\left(P \rightarrow 1\right.$ and $\left.P^{\prime} \rightarrow 1\right)$. Remarkably, such drop happens away from the multicritical points at which the percolation transition and the magnetic transition depart from each other. This implies that, for moderate quantum fluctuations, the magnetic quantum phase transition retains a percolative nature, in full agreement with the picture of quantum percolation, in which the main effect of quantum fluctuations is a spatial renormalization of the percolating cluster. In particular, the correlation length exponent $\nu$ is clearly consistent with the classical value $\nu=4 / 3$ for two-dimensional percolation [2]. The $z$ exponent can also be related to the critical exponents of the classical phase transition. At and around the homogeneous percolation point $P=P^{\prime}=0.5$, where the transition is classical, the exponent $z$ is the scaling dimension of the finite-size gap of the percolating cluster diverging in the thermodynamic limit, $\Delta \sim L^{-z}$. Since the number of sites $N_{c}$ of this cluster diverges as $N_{c} \sim L^{D}$ with $D=91 / 48$ 2], if the finite-size gap vanishes as $\Delta \sim N_{c}^{-\rho}$ it is easy to see that $z=\rho D$. For clusters with the geometry of the chain or of the square lattice, $\rho=1$ [6, 14]. Extending this result to the fractal percolating cluster we obtain $z=D=1.89$.., in agreement with the QMC result.

The concept of quantum percolation has previously been invoked in the context of weakly interacting electronic systems, such as in metal-to-insulator transitions of granular metals 16], and in the integer quantum Hall effect of semiconductor heterostructures 17]. In these examples, quantum mechanics enters mostly through single-particle phenomena, such as localization effects of single-electron wave-functions and quantum tunneling. In contrast, the quantum percolation discussed in the present work occurs in a strongly correlated electron system. Its quantum aspects reside in the formation of local many-body quantum states, i.e. dimer singlet states or RVB states, and it ultimately has the nature of a collective quantum phase transition. The specific example of inhomogeneous bond dilution of the square-lattice Heisenberg antiferromagnet offers a remarkable realization of such scenario, with continuous tuning of the quantum nature of the percolative transition in the system. A continuous modulation of quantum effects at a percolation transition can also be achieved by progressively coupling two square-lattice layers which are site-diluted at exactly the same lattice sites [18. In that case, nonetheless, the estimated critical exponents for the magnetic quantum phase transition differ substantially from those of classical percolation and are much closer to those of a quantum phase transition in a clean system, suggesting that quantum effects alter the percolative nature of the transition.

From the experimental point of view, if different nonmagnetic ions mediate the intra-dimer interactions $(b$ bonds of Fig. [(A)) with respect to the inter-dimer ones $\left(b^{\prime}\right.$ bonds of Fig. 1(A)) in a square lattice antiferromagnet, selective chemical substitution of these two types of ions offers a way to realize inhomogeneous bond disorder. Extensions of the picture given in this paper would be necessary to mimic a realistic experimental situation. Nevertheless, the effects leading to a quantum correction of the percolation threshold should be robust, and they should persist in presence of more general models of inhomogeneous bond disorder.

We acknowledge fruitful discussions with B. Normand, H. Saleur and A. Sandvik. S.H. acknowledges hospitality at KITP, Santa Barbara. This work is supported by the NSF through grant No. DMR-0089882. Computational facilities have been generously provided by the HPCCUSC Center.

[1] P. G. De Gennes, La Recherche (Paris) 7, 919 (1976).

[2] D. Stauffer and A. Aharony Introduction to Percolation Theory. Taylor and Francis, London (1994).

[3] O. P. Vajk et al., Science 295, 1691 (2002).

[4] A. W. Sandvik, Phys. Rev. B 66, 024418 (2002).

[5] S. Sachdev, Quantum Phase Transitions. Cambridge University Press, Cambridge (1999).

[6] S. R. White et al., Phys. Rev. Lett. 73, 886 (1994).

[7] E. Dagotto and T. M. Rice, Science 271618 (1996).

[8] E. Manousakis, Rev. Mod. Phys. 63, 1 (1991).

[9] M. E. J. Newman and R. M. Ziff, Phys. Rev. Lett. 85, 4104 (2000).

[10] O. F. Syljuåsen and A. W. Sandvik, Phys. Rev. E 66, 046701 (2002).

[11] M. Greven and R. J. Birgeneau, Phys. Rev. Lett. 81, 1945 (1998); C. Yasuda et al., Phys. Rev. B 64, 092405 (2001); S. Wessel et al., Phys. Rev. Lett. 86, 1086 (2001).

[12] M. Sigrist and A. Furusaki, J. Phys. Soc. Jpn. 65, 2385 (1996).

[13] S. L. Sondhi et al., Rev. Mod. Phys. 69, 315 (1997).

[14] J. Carlson, Phys. Rev. B 40, 846 (1989).

[15] J. T. Chayes, L. Chayes, D. S. Fisher, and T. Spencer, Phys. Rev. Lett. 57, 2999 (1986).

[16] P. Sheng, Introduction to Wave Scattering, Localization and Mesoscopic Phenomena. Academic Press, Boston, MA (1995).

[17] S. A. Trugman, Phys. Rev. B 27, 7539 (1983).

[18] O. P. Vajk and M. Greven, Phys. Rev. Lett. 89, 177202 (2002); A. W. Sandvik, ibid. 89, 177201 (2002). 